\title{
Personal and Socio-Cultural Barriers to Cervical Cancer Screening in Iran, Patient and Provider Perceptions: a Qualitative Study
}

\author{
Roghieh Bayrami ${ }^{1}$, Ali Taghipour ${ }^{2}$, Hossein Ebrahimipour ${ }^{3,4 *}$
}

\begin{abstract}
Background: Although cervical cancer is preventable and early screening might decrease the associated mortality, challenges faced by the women and health care providers can postpone early detection. This qualitative study aimed to establish patient and provider perceptions about personal and socio-cultural barriers for cervical cancer screening in Mashhad, Iran. Materials and Methods: In the present study, which was conducted in 2012, eighteen participants, who were selected purposefully, participated in individual in-depth, semi-structured interviews, which were recorded, transcribed verbatim, and analyzed using conventional content analysis and Atlas-Ti software. Results: One theme and two categories were derived from data including: cognitive/behavioral factors (lack of a community-based approach to cervical cancer, lack of awareness, wrong attitude and lack of health seeking behaviors) and socio/cultural issues (socio-cultural invasion, mismatch between tradition, modernity and religious, extra marital relationships and cultural taboos). Conclusions: Providing community based approach education programs and employing social policy are needed for preventing of cervical cancer in Iran.
\end{abstract}

Keywords: Cervical cancer - cancer screening - qualitative study - Iran

Asian Pac J Cancer Prev, 16 (9), 3729-3734

\section{Introduction}

Cervical cancer remains a major public health challenge in developing countries. Despite the reduction in the incidence and mortality of cervical cancer in developed countries during the last two decades; developing countries have not been shown such changes in these two indices (Brotto et al., 2008).

Although the cervical cancer incidence rate is low in Iran, nonetheless most cervical cancers are detected at advanced stages where the prognosis is poor (Arbyn et al., 2011). According to the Cancer Registry of the Ministry of Health and Medical Education (MOHME) in Iran, the incidence of cervical cancer has been increased between 2005-2007 (IARC, 2012).

Cervical cancer screening is an essential part of a woman's routine health care; because it can detect cancer or abnormalities that may lead to cervical cancer. A routine Pap smear screening cervical cancer can greatly reduce the mortality risks of cervical cancer (Ebu et al., 2015).Usage of the Pap smear test for cervical cancer prevention would contribute to the access of the Millennium Development Goals through universal access to sexual and reproductive health services to improve women's health (WHO, 2015).
This attainment has resulted in high rates of screening uptake (Group, 2014).

Although cervical cancer is preventable and early screening might decrease the mortality associated with it, most challenges faced by the patients and health care providers can be affected early detection practices (Chirenje et al., 2001; Fort et al., 2011). For example lack of information regarding Pap smear test, lack of a physician recommendation contributes to underuse of Pap, patient fear, the high cost of screening test and restricted access to services are the main barriers for women to perform Pap smear test (Agurto et al., 2004; Waller et al., 2009; Wong et al., 2009). Many studies support the role of some species human papilloma virus (HPV) in cervical cancer (Jafari Shobeiri and Chapari, 2011).

Although the World Health Organization in collaboration with the International Agency for Research on Cancer, offered programs for cervical cancer prevention, the effort should be expanded because the cervical cancer is a multi-factorial disease. According to the apparent differences between the social, economic and sexual activities in Islamic and non-Islamic societies, the considered challenges cannot be generalized to other areas like Iran. For example several studies suggest relationship

${ }^{1}$ Nursing and Midwifery School, ${ }^{2}$ Department of Biostatistics, ${ }^{3}$ Department of Health and Management, Health Sciences Research Center, School of Health, Research Center for Health Services Management, Institute for Futures Studies in Health, Mashhad University of Medical Sciences, Mashhad, Iran *For correspondence: EbrahimipourH@mums.ac.ir 
between religion-related value and cervical cancer screening (Padela et al., 2014). Also lower prevalence of the main risk factors such as onset of intercourse before age 20 years, multiple sexual partners, smoking, and HPV infection have lead to a low incidence of cervical cancer in Muslim countries (Khorashizadeh et al., 2013). On the other hand circumcision among Muslim men is the most likely cause of lower rate of cervical cancer among Muslim women, because circumcision may reduce risk of both acquiring and transmitting Human Papilloma Virus (HPV) (Drain et al., 2006). With respect to these disparities assessing the women's health belief, improving cervical cancer screening, cognition-emotion focused programs and HPV vaccination programs can be appropriate ways for cervical cancer prevention in the Islamic society (Demirtas, 2013) and several Western countries respectively (Khorashizadeh et al., 2013).

Quantitative studies that are occurred with deductive approach based on predetermined topics in the form of questionnaires cannot show actual picture of the experiences of patients and health care workers about cervical cancer (Grove et al., 2012). In order to promote women's health and women's health policies and programs development is required to identifying challenges and gaps in designing programs and offering appropriated services based on the needs and information and scientific evidence (Bayrami et al., 2014). Finding these problems and addressing them is essential for improving the conditions mentioned above.

The current study aims to enhance our understanding of the issues around the widely problems excising about health, cultural and social policies based on the views of health care providers and patients about cervical cancer screening programs, so that eventually our results can be used by policy makers to promote the cervical cancer prevention programs.

\section{Materials and Methods}

\section{Study design, location and population}

This qualitative study was conducted in Mashhad, Iran in 2012. Patients with cervical cancer, gynecology oncology specialists, obstetrics and gynecology specialists, health managers and midwives were selected purposefully from two main sites 1) the Ghaem hospital, the biggest general subspecialty hospital, with specialized services for gynecologic cancers where women had come to utilize the cervical cancer treatment service and 2) Province Health Center where managers with relative experiences were working there. Summary of characteristics of study participants have been shown in Tables 1 and 2 .

The inclusion criterion for patients were recognized cervical cancer. Physicians, midwives and health managers were included in case that they had at least two years of experience in the relevant field.

\section{Procedures}

Sampling was continued until data saturation was achieved-that is, no new codes were present in the datawhich happened at the 18th interview. Interviews took 30 to 100 minutes. The first author conducted individual in-depth, semi-structured interviews. The interviews started using general open-ended questions like: what do you perform pap smear test? (For patients) and what are the barriers to do Pap smear screening? (For provider) Continuing the interview, according to the responses to each of the questions, in-depth probing questions such as 'What do you mean?' 'Why?' 'Explain more' and 'would you please give an example to better convey what you mean?'During the interviews, the researcher recorded nonverbal data such as the facial expression, and position on a special sheet.

\section{Data analysis}

AtlasTi5 software was used for data management. After each interview, data were analyzed using content analysis with a conventional approach. The interviews were transcribed verbatim. The transcribed interviews were read several times. The meaning units related to individual and socio cultural challenges were identified. The codes detected from the participants 'explanations. The codes were classified into subcategories and main categories on the basis of differences and similarities.

\section{Ethics of study}

This study was approved by the Ethics Committee of Mashhad University of Medical Sciences and informed written consent was obtained.

\section{Trustworthiness}

To increase trustworthiness of the data, enough time was allocated for the data collection and the derived codes from each of the interviews were presented to the participants and their views about the meaning of the codes were asked; if their views were inconsistent, their corrective comments were applied. In addition to ensure reliability of coding, the written text of the interviews was presented to some of the external researchers who were not involved in the study and they were asked to check the accuracy of the coding process. No major differences in coding were identified. Minor differences were discussed and resolved between the researchers.

\section{Results}

Overall, one theme and two categories of barriers emerged from data analysis. (Table 1) Each category has been explained in detail subsequently.

\section{Cognitive/behavioral factors}

Lack of community-based approach to cervical cancer: Nowadays medical science faces with different challenges, including social, economic and demographic changes. Regarding to community based education, awareness of the needs of patients and performance of physicians are incremented. Medical education should be move from patient-centered towards the being community base with designing educational programs to improve the health and understanding the health needs of society. Based on the views of participants, there is no community-based approach for cervical cancer preventing programs in our society. 
Perceptions of Patients and Providers of Barriers to Cervical Cancer Screening in Iran: a Qualitative Study

The following quotations from two of the physicians confirm the issue mentioned above: “....... Information must be given to the community. In my opinion education must be started at the health centers with women and continued in society. It must be educated for both women and men at the society. This issue is very important." (Gynecology oncology specialists -3)

Table 1. Characteristics of the Participating Patients

\begin{tabular}{llc}
\hline Variable & \multicolumn{1}{c}{ Participants (n=8) } \\
\hline Education level & Illiterate & 1 \\
& Secondary school & 1 \\
& Diploma & 1 \\
& Bachelor & 3 \\
Employment status & Unemployed & 2 \\
& Employed & 4 \\
& Age Range 34-65 & 4 \\
Age & Mean age (years & $34-65$ \\
& s35 years & 1 \\
& 36-45 years 5 & 2 \\
Stage of cancer & 46-55 years 5 & 3 \\
& 56- 65 years & 2 \\
& Carcinoma in situ & 3 \\
& Stage 1 & 2 \\
& Stage2 & 2 \\
& Stage3 & 1 \\
\hline
\end{tabular}

Table 2. Characteristics of the Participating Health Care Providers

\begin{tabular}{|c|c|c|}
\hline \multicolumn{3}{|c|}{ Health care providers $(\mathrm{n}=10)$} \\
\hline \multicolumn{3}{|l|}{ Occupation } \\
\hline \multicolumn{2}{|l|}{ Midwife } & 3 \\
\hline \multicolumn{2}{|c|}{ Health manager } & 1 \\
\hline \multicolumn{2}{|c|}{ Obstetrics and gynecology specialist } & 3 \\
\hline \multicolumn{2}{|c|}{ Gynecology oncology specialists } & 3 \\
\hline \multicolumn{3}{|l|}{ Age } \\
\hline \multicolumn{3}{|c|}{ Age Range $28-50$} \\
\hline \multicolumn{2}{|c|}{ Mean age (years) } & 46 \\
\hline \multicolumn{3}{|c|}{ Work experience } \\
\hline \multicolumn{2}{|l|}{ Midwife } & 10 years \\
\hline \multicolumn{2}{|c|}{ Health manager } & 12 years \\
\hline \multicolumn{2}{|c|}{ obstetrics and gynecology specialist } & 14 years \\
\hline \multicolumn{2}{|c|}{ Gynecology oncology specialists } & 14 years \\
\hline \multicolumn{3}{|c|}{ Workplace } \\
\hline \multirow{2}{*}{\multicolumn{2}{|c|}{$\begin{array}{l}\text { Midwife } \\
\text { Health manager }\end{array}$}} & health center \\
\hline \multirow{2}{*}{\multicolumn{2}{|c|}{ Health manager }} & province town \\
\hline & & health center \\
\hline \multirow{2}{*}{\multicolumn{2}{|c|}{$\begin{array}{l}\text { Obstetrics and gynecology specialist } \\
\text { Gynecology oncology specialists }\end{array}$}} & private clinic \\
\hline & & $\begin{array}{l}\text { educational, research } \\
\text { and treatment center }\end{array}$ \\
\hline \multicolumn{3}{|c|}{$\begin{array}{l}\text { Table 3. Two Main Categories and Sub-categories of } \\
\text { Barriers to Cervical Cancer Screening }\end{array}$} \\
\hline Category & \multicolumn{2}{|c|}{ Subcategory } \\
\hline $\begin{array}{l}\text { Cognitive/ } \\
\text { behavioral } \\
\text { factors }\end{array}$ & \multicolumn{2}{|c|}{$\begin{array}{l}\text {-Lack of a community-based } \\
\text { approach to cervical cancer } \\
\text {-Lack of awareness } \\
\text {-Wrong attitude } \\
\text {-absence of health seeking behaviors }\end{array}$} \\
\hline $\begin{array}{l}\text { Socio/cul- } \\
\text { tural issues }\end{array}$ & \multicolumn{2}{|c|}{$\begin{array}{l}\text {-Socio cultural invasion } \\
\text {-mismatch between tradition, modernity, and } \\
\text { religious } \\
\text {-extra marital relationship } \\
\text {-cultural taboo }\end{array}$} \\
\hline
\end{tabular}

".... Today the prevalence of many chronic diseases is increasing in our country thus raising public awareness and training among community should be considered." (Specialist doctor in obstetrics and gynecology-3)

Lack of awareness: Public knowledge and perceptions and the social forces that cause and shape health behaviors. Based on the analysis of the participants' comments, generally, the women had a lack of awareness of cervical cancer risk factors and screening behaviors. Confirming the aforementioned subjects, several individuals participating said: "If women has not gynecologic problem it is not necessary to done pap test" (42 years old, cancer patient) "A reason that most women don't take Pap test is they don't accept the Pap test is tool for screening cancer. Women who are asymptomatic and do a Pap smear often have similar ideas. And this shows that awareness about cervical cancer is very low." (Gynecology oncology specialists 2) "Patient doesn't know that genital wart is a STD and it has transmitted from her husband." (Gynecology oncology specialists 1)

Wrong attitude: Attitudes have a powerful influence on behavior. An attitude is an expression of favor or disfavor toward a person, place, thing, or event Majority of patients participating had wrong attitude about cervical cancer and screening test. Iranian are almost all Muslim and their view of health and dead is typically shaped by a combination of traditional and Islamic beliefs, with most believing that God will heal some persons and not others.

One participant said: "I am afraid of Pap test because it may shows that $i$ have cancer also it is painful procedure."(48 years old, cancer patient) Analysis of the interviews showed that the majority of women knew that family history, unhealthy lifestyle and aging were as causing cervical cancer. One participant stated: "As far as I know unhealthy lifestyle can cause types of cancers including cervical cancer. I'm an athlete. I climb. I never thought that someday $i$ will get cervical cancer. I thought only post-menopausal women get cervical cancer."(49 years old, cancer patient)

Negative attitudes can be reflected in one's behavior. So the attitude towards the outcome of a job can be prevented from doing it.One patient told us: "Because of some reason I was afraid of pap testing. I was concerned about clearing cervical cancer by having a Pap smear. I thought that a Pap smear is painful. I thought that they catch up a piece of meat of my uterus and test it."(36 years old, cancer patient).

Lack of health seeking behaviors: Based on the analysis of the participants' comments, the majority of women either never undertook a Pap test prior, or they performed it irregular. Lack of enough knowledge regarding preventable cervical cancer and also embarrassment for genital examination, may lead to lack of health seeking behavior (Sankaranarayanan et al., 2001). These quotations are as evidence to support our claims:

"I am more than willing to go in for screening but doing Pap smear test last much time and so i could not do it regularly. (48 years old, cancer patient)

"Most women because of fear and embarrassment of 
Roghieh Bayrami et al

genital examination don't undertake Pap smear. (midwife 1)

\section{Socio- cultural issues}

Socio cultural invasion: Despite the scientific and informative content of satellite TV programs, these programs do not select based on their scientific applications by youth. They choose the programs which are not cover by the Iranian media. that's why the vulgar and obscene programs are selected by youth and the moral collapse is very high. One participant said: “.... Watching movies via satellite alters sexual relations between young people. Satellite television broadcasts the foreign culture. It introduces living with others instead of promoting the concept of marriage and infidelity in marriage". (Specialist doctor in obstetrics and gynecology-3).

Mismatch between tradition, modernity, and religious: Some conflicts between customary law and Sharia law, intergenerational gaps in the values of religious are the gaps between the values that cause some cultural challenges. These quotations are as evidence to support our claims: "In our culture traditional marriage is carried out among both boys and girls but later we see some of the young girls who have genital wart come to our office. This event represents that sexual relationship before marriage is common in our society." (Gynecology oncology specialists -2)

"In our society, men and boys are more powerful $A$ man; since he is stronger he can have multiple partners. They have no limitations in sex. Men are free to marry ten times. Men always have more freedom in society. But the illegitimate relationship with the opposite sex is forbidden religiously." (Gynecology oncology specialists -2 )

Most participants in this study pointed out that "temporary marriage is another concern. Temporary marriage is a religious act. Iran's sigheh law gives men right to have as many sexual partners simultaneously. Men who have one or several sexual partners can be very problematic. Unfortunately, it is not something to be done."(Specialist doctor in obstetrics and gynecology-2)

Extra marital relationship: Based on the analysis of the participants' comments, the majority of them pointed to increasing of extra marital relationship. "I don't exaggerate, not a day goes and I have not seen a patient with suspected contact in my office. Among the factors that may lead to cancer is HPV; and we disregard to it. Sexual relationships outside the legal marriage cause the cervical cancer. (Gynecology oncology specialists -2)

"Many friendship between boys and girls in our society lead to sex before marriage and finally STD and cervical cancer" (Midwife 2).

Cultural taboo: In the Participants' perspectives the cultural taboos that affect sexual issues lead to sexual themes are keeping quiet in the community with shame of discuss about sexual themes, denial or reservation of breaking sexual norms, and tendency to avoid sexual discourse in the public arena. One of the specialist said that: "women don't like speak about their sexual organs disease even cancers and STDs.

According to the participants' point of view, health and illness beliefs, the role of spirituality/religiosity, the role of family traditions and the role of the community are barriers that can impact the frequency or lack of cervical cancer screenings among our society women. "Discussion of reproductive tract and female genital or sexual transmitted disease is taboo in our culture. Many women become infected by their husband and embarrassment about discussion it even with their physician." "(Specialist doctor in obstetrics and gynecology-1)

"Most families whenever the media want to talk about problems of genital organs Change the channel of media." (Midwife 3). "Embarrassment at exposing reproductive organs during a physical examination may pose a barrier for pap test among Iranian women. Many women feel ashamed the screening procedure. Even many of them find it too invasive." (Gynecology oncology specialists -3)

Cervical cancer is a stigmatized because the cervix is part of the body that most people can not talk about it. "I had not talked with my colleagues about my cervical cancer, they themselves found out that I ill" (52 years old, cancer patient). (.....like the AIDS problem, the media didn't tell about it, finally they sought that all catch it, then they told that say every things about AIDS (Midwife 2).

\section{Discussion}

In this qualitative study some personal and sociocultural barriers of cervical cancer screening in Iran were investigated. Two main categories including: cognitive/ behavioral challenges and socio/cultural challenges emerged from data analysis.

In this study lack of community-based approach to cervical cancer was a challenge facing CCPP. In the present study, clinicians and managers were noted to the need for a community-based approach to CCPP. (Enjezab et al., 2004) have also stated that cervical cancer screening should be considered as a social event and women in the different groups share their experiences together so that barriers such as painful feelings and deepest fears reduce and they know they are not the only person to have this experience. While in poor countries community-based educational programs via mass media and lecture presentations can enhance cervical cancer related information and improve screening behavior (Perkins et al., 2007).

Data analysis showed that low awareness and misconceptions about the screening test, symptoms and etiology of the cervical cancer, would lead to not undertaking Pap smear test. In most study of cervical cancer in Iran and other countries low awareness about Pap smear test was associated with cervical cancer. The results are consistent with our study findings (Wong et al.,2008; Birhanu et al., 2012; Saberi et al.,2012).

In this study, women with cancer had a wrong attitude towards cervical cancer and Pap smear screening method so that many of the women believed that a cancer is 'God's will' which is compatible with other studies findings. The study which was carried out in Somalia reported women's incorrect attitude resulted from traditional and 
religious belief among people (Abdullahi et al., 2009).

Beliefs that a genital tract should be seen by a husband and a midwife, furthermore, putting the metal speculum is a painful procedure are the obstacles for pap smear test in Kenya (Kivuti et al., 2013). In another studies women had a positive attitude towards screening method that is in contrast with the results of our study (Claeys et al., 2002; Huchko et al ., 2011). Demographic, cultural and process factors may be associated with negative or positive attitudes. Due to the constraints mentioned, there is an obvious need for adequate training of women for solution barriers and strengthening incentives of women for doing screening cancer test.

The majority of patients in this study, either never undertook a Pap test before, or they performed it irregular which is compatible with other studies findings in Iran. In the study that conducted in Isfahan $87.7 \%$ of 324 patients had at least one Pap smear test, of whom $38.7 \%$ had the test done every three years and $50.7 \%$ had done irregularly also most women in Kashan had not repeat Pap smear test at standard intervals (Saberi et al., 2012). This is important, because the most important factors in determining program success is coverage of the target population by screening test (Wongwatcharanukul et al., 2014).

Cultural invasion is one of the challenges associated with CCPP. Satellite network tries to show normal couple betray to each other. Also it shows normal male and female sex before marriage and abortion for girls as a natural solution to relieve the anxieties of possible normal sexual relations. Satellite Promoting the concept of living with others rather than marriage and thus spread STD and AIDS and cervical cancer consequently.

The majority of participants in this study pointed out that temporary marriage and relationships outside of marriage are the main challenges related to CCPP. Mortazavi and his collages said that the prevalence of HPV in cervical carcinomas in Iran is similar to those reported in other countries. It appears that HPV-16 is the most common type associated with cervical cancer in Iran (Mortazavi et al., 2002).

The physicians and midwives participating in this study believed that having multiple sex partners or outside of marriage relationship are the risk factors for HPV and consequently cervical cancer. These findings are consistent with results of other studies (Hanley et al., 2014; De Gonzalez et al., 2004; Jafari Shobeiri, 2007). Results of studies have shown that young men and women are at higher risk for HPV infection (Naggar et al., 2010) since the temporary marriage, is not restricted to one sexual partner, and one can have multiple sex partners increase incidence of sexually transmitted diseases, including HPV.

With inadequate knowledge of the current state about sexually transmitted diseases and sexual health, full attention should be given to the promotion of condom use and sexual health.

According to the participants view in this study mismatch between tradition, modernity, and religion was one of the challenges facing the CCPP. Latifnejad and her colleagues have pointed to social and cultural challenges in sex education (Nejad et al., 2000) So the influence of
Western culture on youth as consequences of modern communication technology result in that might have challenge with the traditions; fascinated by the West and leave himself from constraints of tradition and religion.

Among the socio-cultural challenges associated with CCPP is a cultural taboo. Cancer in terms of cultural, individual, social, economic and political status has a particular importance. Now the cancer is a taboo in our society, in public opinion and even specialists. Cancer equals with deaths. And this, at least in some cases led to failure in the proper care and treatment.

Even in the radio and television programs are not discussed about reproductive cancers and STDs. The results of a study showed that only $2 \%$ of women had been encouraged by radio and television for Pap smear test (Tabeshian and Firozeh, 2009). While with primary prevention, as at least one third of cancer cases are preventable. If the correct and proper screening test is performed this figure will reach $80 \%$.

The results of this study indicate that women face cultural, emotional and practical barriers to do Pap smear test. In other studies, major barrier to routine screening tests is socio-cultural barriers such as shame of pelvic examination (Sankaranarayanan et al., 2008).

In conclusion, cognitive/behavioral and socio/cultural challenges are two categories that lead to increasing cervical cancer in Iran. It is desirable to provide health and wellbeing community based approach education programs and employing social policy for preventing of cervical cancer. Regarding study limitations, in a qualitative study purposive sampling and small number of participants are considered as limitation for study. Hence generalization of the results is limited.

\section{Acknowledgements}

This article is part of a study which had financial support of Deputy of Research of Mashhad university of Medical Sciences (Grant No 910380).We are also grateful of all participating in this project. Authors of the manuscript declare that they have no conflict of interest.

\section{References}

Abdullahi A, Copping J, Kessel A, Luck M, Bonell C (2009). Cervical screening: perceptions and barriers to uptake among somali women in Camden. Public Health, 123, 680-5.

Agurto I, Bishop A, Sanchez G, Betancourt Z, Robles S (2004). Perceived barriers and benefits to cervical cancer screening in Latin America. Prev Med, 39, 91-8.

Al-Naggar RA, Low W, Isa ZM (2010). Knowledge and barriers towards cervical cancer screening among young women in Malaysia. Asian Pac J Cancer Prev, 11, 867-73.

Arbyn M, Castellsague X, de Sanjose S, et al (2011). Worldwide burden of cervical cancer in 2008. Ann Oncol Dec, 22, 2675-86.

Bayrami R, Taghipour A, Ebrahimipour H (2014). Challenges of providing cervical cancer prevention programs in iran: a qualitative study. Asian Pac J Cancer Prev, 15, 10071.

Birhanu Z, Abdissa A, Belachew T, et al (2012). Health seeking behavior for cervical cancer in Ethiopia: a qualitative study. Int J Equity Health, 11, 83. 
Brotto L, Chou A, Singh T, Woo J (2008). Reproductive health practices among indian, indo-canadian, canadian east asian, and euro-canadian women: the role of acculturation.J Obstet Gynaecol Can, 30, 229.

Chirenje ZM, Rusakaniko S, Kirumbi L, et al (2001). Situation analysis for cervical cancer diagnosis and treatment in east, central and southern African countries. Bull WHO, 79, 127-32.

Claeys P, Gonzalez C, Gonzalez M, (2002). Determinants of cervical cancer screening in a poor area: results of a population based survey in Rivas, Nicaragua. Trop Med Int Health, 7, 935-41.

De Gonzalez AB, Sweetland S, Green J(2004). Comparison of risk factors for squamous cell and adenocarcinomas of the cervix: a meta-analysis. Br J Cancer, 90, 1787-91.

Demirtas B (2013). Review of strategies in promoting attendance for cervical screening. Asian Pac J Cancer Prev, 14, 3263-7.

Drain PK, Halperin DT, Hughes JP, et al.(2006). Male circumcision, religion, and infectious diseases: an ecologic analysis of 118 developing countries. BMC Infect Dis, $\mathbf{6}, 172$.

Ebu NI, Mupepi SC, Siakwa MP, Sampselle CM (2015). Knowledge, practice, and barriers toward cervical cancer screening in Elmina, Southern Ghana. Int J Womens Health, 7, 31 .

Enjezab B, Faraj Khoda T, Mojahed S, Bokaei M (2004). Barriers and motivators related to cervical and breast cancer screening. J Shahid Sadoughi University Med Sci.

Farajzadegan Z, Nourbakhsh SF, Mostajeran M, Loghmani A (2012). Cervical cancer screening status in 35 to 60 year-old women in Isfahan, Iran. J Isfahan Med School, 30, 1542-49.

Fort VK, Makin MS, Siegler AJ, Ault K, Rochat R(2011). Barriers to cervical cancer screening in Mulanje, Malawi: a qualitative study. Pat Prefer Adherence, 5, 125.

Group GH (2012). Integrating Cervical Cancer Screening Into HIV Services in Sub-Saharan Africa. [cited 2015 1/1]; Available from: http://globalhealthsciences.ucsf.edu/sites/ default/files/content/ghg/e2pi-integrating-cervical-cancer. pdf.

Grove SK, Burns N, Gray J (2012). The practice of nursing research: appraisal, synthesis, and generation of evidence: Elsevier Health Sciences.

Hanley SJB, Yoshioka E, Ito Y, et al (2014). An exploratory study of japanese fathers' knowledge of and attitudes towards HPV and HPV vaccination: does marital status matter? Asian Pac J Cancer Prev, 15, 1837-43.

Huchko MJ, Bukusi EA, Cohen CR (2011). Building capacity for cervical cancer screening in outpatient HIV clinics in the Nyanza province of western Kenya. Int J Gynecol Obstet, 114, 106-10.

International Agency for Research on Cancer (2012). Estimated Incidence, Mortality and Prevalence Worldwide in 2012. Available at: http:// globocan.iarc.fr. Accessed on 2014 march, 1 .

Jafari Shobeiry M, Chapari Ilikhchi A (2010). Role of demographic and socioeconomic factors in cervical cancer. Iranian J Obst Gynecol Infertility, 13, 22-31.

Jafari Shobeiri M (2007). Screening for cervical cancer and precancerous lesions in Tabriz. Med J Islamic Republ Iran, 21, 1-10.

Kivuti-Bitok LW, Pokhariyal GP, Abdul R, McDonnell G (2013). An exploration of opportunities and challenges facing cervical cancer managers in Kenya. BMC Res Notes, $6,136$.

Khorasanizadeh F, Hassanloo J, Khaksar N, et al(2013). Epidemiology of cervical cancer and human papilloma virus infection among Iranian women-Analyses of national data and systematic review of the literature. Gynecol oncol, 128, 277-81.
Mortazavi S, Zali M, Raoufi M (2000). The prevalence of human papillomavirus in cervical cancer in Iran. Asian Pac J Cancer Prev, 3, 69-72.

Padela AI, Peek M, Johnson-Agbakwu CE, et al (2014). Associations between religion-related factors and cervical cancer screening among muslims in Greater Chicago. J Low Genit Tract Dis, 18, 326-32.

Perkins RB, Langrish S, Stern LJ, Simon CJ (2007). A community-based education program about cervical cancer improves knowledge and screening behavior in Honduran women. Revista Panamericana de Salud Publica, 22, 187 93.

Roudsari RL, Javadnoori M, Hasanpour M, Hazavehei SMM, Taghipour A (2013). Socio-cultural challenges to sexual health education for female adolescents in Iran. Iran $J$ Reprod Med, 11, 101.

Saberi F, Sadat Z, Abedzadeh M (2012). Factors associated with cervical cancer screening and its barriers among women: Kashan, Iran. Payesh, 11, 365-70.

Sankaranarayanan R, Budukh AM, Rajkumar R (2001). Effective screening programmes for cervical cancer in low-and middle-income developing countries. Bulletin World Health Organizat, 79, 954-62.

Sankaranarayanan R, Thara S, Esmy PO, Basu P(2008). Cervical cancer: screening and therapeutic perspectives. Med Princ Pract, 17, 351-64.

Tabeshian A, Firozeh F (2009). The effect of health education on performing Pap smear test for prevention of cervix cancer in teachers of Isfahan city. Med Sci J Islamic Azad UnivesityTehran Med Branch, 19, 35-40.

Waller J, Bartoszek M, Marlow L, Wardle J (2009). Barriers to cervical cancer screening attendance in England: a population-based survey. J Med Screen, 16, 99-204.

WHO (2015). Sexual and reproductive health [cited 2015 1/1]; August 18, 2014 marked the 500-day milestone until the target date to achieve the Millennium Development Goals (MDGs), Available from: http://www.who.int/ reproductivehealth/topics/mdgs/en/.

Wong L, Wong Y, Low W, et al (2009). Knowledge and awareness of cervical cancer and screening among Malaysian women who have never had a Pap smear: a qualitative study. Singapore Med, 50, 49-53.

Wongwatcharanukul L, Promthet S, Bradshaw P, Jirapornkul C, Tungsrithong N (2014). Factors affecting cervical cancer screening uptake by Hmong hilltribe women in Thailand. Asian Pac J Cancer Prev, 15, 3753.

Wong L, Wong Y, Low W, Khoo E, Shuib R (2008). Cervical cancer screening attitudes and beliefs of Malaysian women who have never had a pap smear: a qualitative study. Int $J$ Behav Med, 15, 289-92. 